DR. DAVID L STOCUM (Orcid ID : 0000-0002-8523-744X)

Article type : Review Article

\title{
Nerves and Proliferation of Progenitor Cells in Limb Regeneration
}

\author{
Running title: Nerves and limb regeneration
}

David L. Stocum

Department of Biology

Indiana University-Purdue University Indianapolis

Indianapolis, IN

Address: David L. Stocum

Department of Biology

723 W. Michigan St.

Indiana University-Purdue University Indianapolis

Indianapolis, IN 46202

Email: dstocum@iupui.edu

This is the author's manuscript of the article published in final edited form as:

Stocum, D. L. (2018). Nerves and Proliferation of Progenitor Cells in Limb Regeneration. Developmental Neurobiology, 


\section{Abstract}

Nerves, in conjunction with the apical epidermal cap (AEC), play an important role in the proliferation of the mesenchymal progenitor cells comprising the blastema of regenerating urodele amphibian limbs. Reinnervation after amputation requires factors supplied by the forming blastema, and neurotrophic factors must be present at or above a quantitative threshold for mitosis of the blastema cells. The AEC forms independently of nerves, but requires nerves to be maintained. Urodele limb buds are independent of nerves for regeneration, but innervation imposes a regenerative requirement for nerve factors on their cells as they differentiate. There are three main ideas on the functional relationship between nerves, AEC and blastema cells: (1) nerves and AEC produce factors with different roles in maintaining progenitor status and mitosis; (2) the AEC produces the factors that promote blastema cell mitosis, but requires nerves to express them; (3) blastema cells, nerves, and AEC all produce the same factor(s) that additively attain the required threshold for mitosis.

\section{Introduction}

Peripheral nerves, along with neurons of the central nervous system (CNS), blood vessels, immune cells, and endocrine organs, have historically been viewed as part of an integrated signaling system that regulates the biochemical and biomechanical functions of the body. More recently, nerves have been shown to play important roles in tissue and appendage regeneration in invertebrates and vertebrates, and in the progression of some cancers by stimulating the growth of stem and progenitor cells (reviewed by Kumar and Brockes, 2012; Boilly et al, 2017). A widely used experimental model to study the role of nerves in regeneration is the regenerating urodele limb (Dinsmore, 1991, 1998). The present review summarizes recent research results and ideas on how 
nerves and the wound epidermis promote the proliferation of progenitor cells in regenerating urodele amphibian limbs, and on the identification of the molecular agents involved.

\section{Formation of the Regeneration Blastema}

Urodele limbs regenerate via a blastema of undifferentiated progenitor cells released by the proteolytic histolysis of mesodermal limb tissues. These cells aggregate under a wound epidermis that is thickened into a ridge called the apical epidermal cap (AEC). There are two mechanisms of tissue contribution, by the release of resident progenitor cells from muscle, periosteum and perhaps as yet undefined fibroblast populations, and the dedifferentiation of nucleated myofiber fragments, fibroblasts, and Schwann cells (reviewed by Stocum, 2017). The proportion of regenerate muscle contributed by release of resident progenitors (satellite cells) and by dedifferentiation (myofiber fragmentation) varies with species and developmental status (Sandoval-Guzman et al, 2014; Tanaka et al, 2016). The contribution of each tissue to the blastema also varies, with dermal fibroblasts contributing over half the blastema cells and cartilage contributing little or none (Muneoka et al, 1986; McCusker et al, 2016). Redifferentiation is lineage-specific except for blastema cells derived from dermal fibroblasts, which differentiate not only into new fibroblasts, but also transdifferentiate into the new chondrocytes of the skeletal elements, congruent with the minimal contribution of cartilage to the blastema (Kragl et al, 2009). The wound epidermis/AEC does not contribute to the progenitor cell population, but redifferentiates as epidermis. Figure 1 illustrates the phases and stages of urodele limb regeneration.

\section{Role of the nerve in blastema cell proliferation}

In the 1940s, a series of denervation studies by Singer and colleagues on regenerating newt limbs revealed that a threshold quantity of axons per unit area of limb tissue is required for regeneration.

This article is protected by copyright. All rights reserved. 
Singer synthesized his results into the neurotrophic hypothesis (Singer, 1952, 1964, 1965), which states that sensory, motor and autonomic nerves provide the early blastema with a quantitative threshold of neurotrophic factor(s) required for blastema growth. The normal sensory innervation, but not the motor or sympathetic innervation, is sufficient to meet the threshold requirement. There is no qualitative difference in the ability of nerve type to promote regeneration, for if the motor nerve supply is augmented by deviating motor nerves such that they could branch into denervated sensory endoneurial tubes, regeneration takes place (Sidman and Singer, 1960; Thornton, 1960).

The nerve is not required for histolysis and the initial accumulation of blastema cells, but is essential for blastema cell proliferation. Blastema cells enter the cell cycle and synthesize DNA after denervation of an amputated limb (Mescher and Tassava, 1976), but mitotic index is reduced to zero by denervation at any stage of blastema formation (Goldhamer and Tassava, 1987). Once the blastema has grown to a critical size, it becomes independent of the nerve for differentiation and morphogenesis, but remains nerve-dependent for blastema cell mitosis, as evidenced by the fact the blastema forms a miniaturized regenerate (reviewed by Thornton, 1968; Stocum and Cameron, 2011; Pirotte et al, 2015; Boilly et al, 2017, Stocum, 2017). Regeneration of axons following amputation is stimulated by factors expressed by blastema cells, as shown by in vitro co-culture experiments (Richmond and Pollack, 1983; Boilly and Bauduin, 1988; Bauduin et al, 2000). Several known neurotrophic factors such as brain-derived neurotrophic factor (BDNF), neurotrophins 3 and $4(\mathrm{NT3}, 4)$, glial derived neurotrophic factor (GDNF) and hepatocyte growth factor/scatter factor (HGF/SF) can substitute for blastema tissue in promoting axon outgrowth in vitro (Tonge and Leclere, 2000). These factors are produced by Schwann cells and promote neuron survival and axon outgrowth in the regenerating peripheral nerves of mammals (reviewed by Fu and Gordon, 1997), raising the question of whether they might be produced by the subpopulation of blastema cells derived from Schwann cells. Retinoic acid has been shown to be produced by the blastema of the

This article is protected by copyright. All rights reserved. 
regenerating newt limb, and to induce outgrowth of spinal cord axons toward the blastema via the RAR $\beta$ receptor (Dmetrichuk et al, 2005). Regardless, axon outgrowth is significantly more vigorous with blastema tissue, suggesting that blastema cells produce other, as yet unidentified, factors that encourage neuron survival and axon outgrowth. A comparison of genes expressed by axolotl dorsal root ganglia (DRG) cells in the presence and absence of blastema cells revealed 27 DRG genes that were differentially expressed in the presence of blastema cells (Athippozhy et al, 2014). Figure 2 depicts the relationship between nerves and blastema growth, and also the influence of blastema cells on regenerating axons.

Recently, studies of zebrafish caudal fin regeneration have defined a role for $\mathrm{H}_{2} \mathrm{O}_{2}$, a reactive oxygen species (ROS), in regeneration (reviewed by Meda et al, 2017). Injured nerves produce high levels of $\mathrm{H}_{2} \mathrm{O}_{2}$ through activation of the Hedgehog (Shh) pathway. The $\mathrm{H}_{2} \mathrm{O}_{2}$ appears to provide an environment conducive to blastema formation and also stimulates axon regrowth. Denervation prevents this production and inhibits regeneration. ROS production has not as yet been investigated in the nerves of regenerating amphibian limbs. Macrophages are essential for blastema formation in regenerating amphibian limbs (reviewed by Mescher, 2017), and produce bactericidal $\mathrm{H}_{2} \mathrm{O}_{2}$ during mammalian skin wound repair that might also contribute to axon regeneration and other aspects of wound repair and blastema formation (reviewed by Clark, 1996, Stocum 2017).

Transcriptomic analysis has revealed that expression of genes specific to wound repair or muscle regeneration do not differ in amputated control and denervated limbs, but transcription of genes regulating proliferation is rapidly reduced in denervated limbs coincident with the start of blastema growth (Monaghan et al, 2009). This is reflected in a $50-70 \%$ reduction of protein synthesis after denervation without any effect on the amino acid precursor pool, rate of protein degradation, or

This article is protected by copyright. All rights reserved. 
rate of translation (Dresden, 1969; Lebowitz and Singer, 1970; Manson et al, 1976; Choo et al, 1978). Changes in the protein profile are quantitative (Singer and Ilan, 1977; Singer, 1978) and are particularly notable in the synthesis of extracellular matrix (ECM) proteoglycans, collagen, and collagen-associated glycosaminoglycans (GAGs). Hyaluronate is the major GAG synthesized during blastema formation in adult newt limbs (Smith et al, 1975) and is reduced the most by denervation (Mescher and Munaim, 1986; Young et al, 1989). The effect of denervation on the regulation of a wide array of individual genes and proteins revealed by global transcriptomic and proteomic analysis of regenerating amphibian limbs (Monaghan et al, 2009; Looso et al, 2013; Rao et al, 2009, 2014; Voss et al, 2015) can now be investigated, facilitated by the sequencing, assembly, and analysis of the axolotl genome (Nowoshilow et al, 2018) coupled with CRISPR (clustered regularly interspersed short palindromic repeats) gene editing technology (Charpentier and Doudna, 2013; Fei et al, 2014, 2016; Flowers et al, 2014).

\section{Role of the AEC in blastema cell proliferation}

The apical ectoderm of anuran, avian, and reptilian limb buds is thickened into a cap called the apical ectodermal ridge (AER) that is indispensable for outgrowth of the limb bud mesoderm via a reciprocal mesenchymal (Fgf10): epithelial (Fgf8) induction (Gilbert and Barresi, 2016). The apical ectoderm of urodele limb buds does not form an AER, but it has the same crucial outgrowthpromoting function (reviewed by Stocum, 1975, 1995). However, the wound epidermis of the regenerating urodele limb is configured within a few days after amputation into the AEC that is rich with sensory innervation and is essential for blastema cell proliferation (reviewed by Thornton, 1968; Stocum, 2017).

The AEC synergizes with the nerve to promote the mitosis of mesenchymal progenitor cells. Endo et al (2004) showed that the initial formation of the AEC is independent of nerves, but that its

This article is protected by copyright. All rights reserved. 
maintenance is nerve-dependent. Other experiments have shown that preventing contact between the wound epidermis or AEC and underlying tissues after amputation leads to regenerative failure, even in the presence of innervation (Mescher, 1976; Chew and Cameron, 1983; reviewed by Stocum and Cameron, 2011; Stocum, 2017). Blastemas deprived of the AEC during their growth phase and implanted into a dorsal fin tunnel such that they cannot contact wound epidermis form miniature regenerates, but unlike denervated blastemas, these regenerates are also truncated distally (Stocum and Dearlove, 1972). Positioning the mesenchyme so that its distal tip protrudes from the tunnel and is re-covered by fin wound epidermis also produces a miniature regenerate, but one that is complete in the PD axis, as in denervated limbs (Fig. 3). In these experiments, the blastema mesenchyme is also denervated, but innervation by nerves of the dorsal fin is likely to be quantitatively insufficient. Cell proliferation was not directly assessed in these experiments, but miniaturization again suggests that the AEC plays a role in mitosis. More direct evidence for a mitogenic role of the AEC is that there is a 3-4 fold reduction in DNA synthesis and mitosis of AECfree blastema cells cultured in vitro transfilter to dorsal root ganglia or with brain neurons (Globus et al, 1980; Smith and Globus, 1989) (Fig. 3). The molecular effects of AEC deprivation on subjacent blastema cells have not been assessed, but 125 genes that are highly up regulated in the AEC have been identified by transcriptome analysis (Campbell et al, 2011).

\section{Hypotheses Regarding the Functional Relationship of Nerve and AEC in Blastema Cell Proliferation}

Three major ideas have been put forward as to the nature of the nerve: AEC functional relationship in promoting blastema cell proliferation (Stocum, 2017) (Fig. 4). These are (1) the nerve and AEC provide separate factors with different roles in the cell cycle; (2) the AEC provides all factors necessary for the cell cycle, but is nerve-dependent to express them; (3) multiple tissues all express the same mitogen that drives blastema growth.

This article is protected by copyright. All rights reserved. 


\section{Nerve and AEC have different but complementary roles in the cell cycle}

The results of experiments on DNA synthesis and mitosis by blastema cells in innervated vs. denervated axolotl and newt limbs have suggested different but synergistic roles for nerves and AEC in the blastema cell cycle (reviewed by Tassava and McCullough, 1978). Labeling of amputated limbs deprived of nerves or wound epidermis with $\left[{ }^{3} \mathrm{H}\right]$-thymidine showed that DNA synthesis in nascent blastema cells is independent of both these tissues, but that in the absence of either one the labeled cells were arrested in $\mathrm{G}_{2}$, undergo apoptosis and are removed by macrophages (Mescher and Tassava, 1976; Loyd and Tassava, 1980; Mescher et al, 2000; Yun et al, 2015). These results led Tassava and Mescher (1975) to propose that injury stimulates blastema cells to enter the cell cycle and that the AEC maintains the cells in an undifferentiated state that renders them responsive to mitogenic signals supplied by the nerve. This idea is consistent with the results of the in vitro transfilter experiments by Globus et al (1980) and Smith and Globus (1989) in which adult newt blastema cells grown opposite dorsal root ganglia or brain cells fail to undergo mitosis in the absence of the wound epidermis and withdraw from the cell cycle, and differentiate as cartilage, whereas in the presence of both epidermal cells and neural tissue, they are maintained in an undifferentiated state and proliferate.

\section{The AEC is dependent on the nerve to express blastema cell mitogens}

The neural requirement for regeneration is imposed on the urodele limb bud only as it becomes innervated at late stages of its development (Fekete and Brockes, 1988). Urodele limbs rendered aneurogenic by extirpating the neural tube during early embryogenesis do not acquire nervedependence for regeneration (Yntema, 1959a, b), but presumably remain dependent on the wound AEC for blastema cell mitosis, suggesting that innervation does not alter the requirement of blastema cells for mitogenic factors, but rather decreases the capacity of the AEC to provide them.

This article is protected by copyright. All rights reserved. 
Thus, a second hypothesis to explain blastema cell proliferation in both aneurogenic and neurogenic limbs is that the AEC provides diffusible mitogens, but in neurogenic limbs nerve axons impose a dependency for their trophic factors on the AEC to express them (Stocum, 2011). This hypothesis is also compatible with the in vitro experiments of Globus et al (1980).

\section{Multiple tissues produce the same mitogenic factor}

Nerve dependence/independence for regeneration can be oscillated back and forth. Nerve dependence of aneurogenic larval limbs can be instituted by transplanting them to neurogenic larvae. If the limbs are then denervated for a period of time, they can regain nerve independence (Thornton and Thornton, 1970). Even adult newt limbs showed some capacity for nerveindependent regeneration when maintained in a denervated condition after grafting them to the back (Singer and Mutterperl, 1963). Singer (1965) explained the ability of limb buds and differentiated aneurogenic limbs to regenerate by postulating that all their tissues express the neurotrophic factor. Production of the factor is suppressed and is taken over by axons as they innervate the developing limb bud or aneurogenic limb. Thus, the nerve itself forces changes to the differentiating tissues of the urodele limb that render the progenitor cells of the blastema nervedependent.

\section{Candidate AEC and neural factors}

Considering all three models, candidates to be AEC and neural factors should meet several criteria (Brockes, 1984). First, they should be expressed by the AEC or dorsal root ganglion (DRG) cell bodies. Further criteria to be an AEC mitogen are expression of the mitogen's receptor in the blastema mesenchyme, loss of mitogen expression by denervation, ability of the mitogen to support regeneration of denervated or AEC-deprived limbs from early blastema formation to digit stages,

This article is protected by copyright. All rights reserved. 
and expression of the mitogen by the AEC of regenerating aneurogenic limbs. Neural factors should be transported from DRG cell bodies along limb sensory nerve axons to the AEC where they bind to their receptor, denervation should prevent blastema cell mitosis by abolishing expression of AEC factors, and the nerve candidates should support regeneration to digit stages in denervated limbs.

Candidate AEC factors: Fibroblast growth factors and the anterior gradient protein (AGP) are candidates expressed by the AEC in vivo (Christensen et al, 2001; 2002; Han et al, 2001; Kumar and Brockes, 2007). Blastema cells express the bek (FGFR2) receptor for Fgfs (Poulin et al, 1993; Poulin and Chiu, 1995) and the AGP receptor Prod1 (Kumar and Brockes, 2007). Fgf1 elevates the mitotic index of cultured blastema cells (Albert et al, 1987; Albert and Boilly, 1988; Boilly et al,1991), and Fgf2 elevates the mitotic index of blastema cells in amputated limbs covered by full-thickness skin (Chew and Cameron, 1983). The only AEC candidate factors so far reported to be down regulated in vivo by denervation and to substitute for the nerve in supporting the regeneration of denervated limbs to digit stages are Fgf2 (Mullen et al, 1996) and AGP (Kumar and Brockes, 2007). Fgf2 was administered in beads only to late stage blastemas.

AGP is strongly expressed in the Schwann cells surrounding the axons of regenerating newt limbs at 5 and 8 days post-amputation, when histolysis and initial dedifferentiation is under way. By 10 days post-amputation, AGP expression shifts to the gland cells of the AEC, coincident with formation of the accumulation blastema. Denervation abolishes AGP expression by Schwann cells and in the AEC, indicating its dependence on axons. The AGP gene supports regeneration to digit stages when electroporated into denervated newt limbs five days post-amputation. Conditioned medium of Cos7 cells transfected with the AGP gene stimulates BrdU (bromodeoxyuridine) incorporation into cultured blastema cells. Incorporation is blocked by antibodies to Prod1, suggesting that AGP acts

This article is protected by copyright. All rights reserved. 
directly on blastema cells through Prod1 to stimulate their proliferation (Kumar and Brockes, 2007). Finally, AGP is expressed by the AEC of regenerating aneurogenic limbs (Kumar et al, 2011), supporting the idea that AGP is a blastema cell mitogen that requires neurotrophins to be expressed in regenerating neurogenic limbs. Whether Fgf2 is expressed in the aneurogenic AEC has not been investigated.

Nerve candidate factors: Factors expressed by DRG neurons that promote blastema cell proliferation in vitro include transferrin (Mescher and Kiffmeyer, 1992; Mescher et al, 1997), substance P (Globus and Alles, 1990; Globus et al, 1991), and Fgf2 (Mullen et al, 1996). Combinations of Fgf8 and BMP (bone morphogenetic protein) have also been tested as neurotrophic factors. Both are expressed in DRG neurons and are detectable in peripheral limb nerve axons in vivo (Satoh et al, 2016). Furthermore, they can substitute for the nerve in the outgrowth of a supernumerary axolotl limb (Makanae et al, 2014). There is now evidence that glial growth factor 2 (Ggf2, neuregulin) (Law et al, 2004), is produced by nerves and blastema cells during blastema growth. Neuregulin is mitogenic for Schwann cells (Davies, 2000) and was suggested over 30 years ago as a nerve factor for limb regeneration (Brockes, 1984; Brockes and Kintner, 1986). It is expressed by DRG neurons, is present in the blastema, and is decreased by denervation. A newt clone of the Ggf2 gene was briefly mentioned to rescue regeneration to digit stages in denervated axolotl limbs when injected intraperitoneally during blastema formation (Wang et al, 2000).

A more detailed study of neuregulin 1 (NRG1) in regenerating axolotl limbs (Farkas et al, 2016) showed that transcripts of $n r g 1$ and its receptors erbb2 and erbb3 are expressed by the basal cells of the AEC and by $56 \%$ of the blastema mesenchyme cells. Antibody staining revealed expression of NRG1 and ErbB2 in dorsal root ganglia and peripheral limb nerves. Denervation of 16-day blastemas

This article is protected by copyright. All rights reserved. 
decreased the number of $n r g 1$-expressing mesenchymal blastema cells by $26 \%$. Western blotting for NRG1 shows only a slight drop in intensity in denervated blastemas, but the percentage of BrdU+ cells co-localizing with NRG1 was diminished by $20 \%$, a statistically significant reduction. Inhibition of NRG1/ErbB2 signaling by immersion of animals in mubritinib abolished blastema formation in amputated innervated limbs. Treatment of 16-day innervated blastemas resulted in miniature regenerates, equivalent to the regenerates obtained by delaying denervation until a well-established blastema has formed. NRG1-soaked beads implanted under the wound epithelium of denervated limbs at seven days post-amputation induced blastema formation. Bead implants every four days from 19-36 days post-amputation supported regeneration to digit stages, though not to the same degree as in innervated controls.

These results support Singer's neurotrophic hypothesis in which multiple limb tissues have the capacity to produce a mitogenic factor essential for blastema cell mitosis. They suggest a synergistic relationship between nerve and blastema cells in which blastema cells autonomously express NRG1 in the absence of nerve, but at a level that is insufficient for mitosis. NRG1 from motor neurons would stimulate blastema cells destined to form Schwann, skeletal and muscle cells to increase their own NRG1 expression. The nerve and blastema cells would thus work synergistically to express a single molecule, NRG1, to attain a threshold level sufficient for mitosis. Although the effect of denervation on NRG1 expression by the AEC was not reported by Farkas et al (2016), expression of NRG1 by the AEC could be incorporated into the model by postulating that NRG1 expression by sensory innervation would stimulate NRG1 production by the AEC for diffusion into the underlying mesenchymal cells. This kind of synergism would explain why increasing motor innervation in the absence of sensory innervation enables complete regeneration, because the required threshold level of NRG1 could be reached in the absence of sensory nerves. The addiction to nerve for regeneration

This article is protected by copyright. All rights reserved. 
that arises during limb development is thus interpreted as a nerve-induced quantitative increase in the requirement by blastema cells for NRG1, which conforms to Singer's original ideas.

In any event, this model predicts that (1) sensory innervation alone can maintain the threshold expression of NRG1, whereas sensory denervation will reduce the expression of NRG1 below threshold; and (2) augmenting motor innervation in the absence of sensory innervation will maintain the threshold expression of NRG1. Experiments to test these predictions will hopefully be undertaken, and to investigate the AEC as another possible source of NRG1.

Several questions remain about the synergistic relationship between nerve, AEC, and blastema cells that require further research. (1) Can we label AEC and neural candidate factors and show that they move into the blastema and bind to receptors on blastema cells? (2) Are neural factors such as NRG1 and Fgf2 expressed in limb buds or the blastemas of amputated aneurogenic limbs? (3) If they are not, does exogenous administration of these factors render aneurogenic limbs nerve-dependent? Another relevant question is whether the epithelial: mesenchymal interaction that characterizes urodele limb bud development and aneurogenic limb regeneration is maintained in the regeneration of neurogenic limbs or is completely replaced by a nerve: AEC synergy. Does the blastema mesenchyme produce a non-neural factor such as Fgf10 necessary to maintain the AEC in addition to neural factors? Growth factor-mediated epithelial: mesenchymal interaction in urodele limb bud development and regenerating aneurogenic limbs has not been sufficiently investigated. The expression of AGP in aneurogenic limbs suggests that AGP might be part of an epithelial: mesenchymal interaction in urodele limb bud development. Fgf8 and Fgf10 are both expressed in urodele limb buds and regeneration blastemas of neurogenic limbs (Han et al, 2001; Christensen et

This article is protected by copyright. All rights reserved. 
al, 2001), but it has not been shown that expression of these growth factors is interdependent as it has for Xenopus limb bud development and limb regeneration (Yokoyama et a, 2000; 2001).

\section{Conclusion}

Progenitor cells of the regeneration blastema in urodele amphibians produce factors that support the regeneration of axons into the blastema. In turn, nerves, in conjunction with the AEC, produce neurotrophic factors essential for the mitosis of progenitor cells during growth of the blastema. There are three main hypotheses on how these two tissues interact to drive blastema growth, but the available evidence fits all three. Further experimentation will be required before favoring one idea over another. Elements of all three hypotheses may be required to explain how the proliferation of blastema progenitor cells is promoted during limb regeneration, and there might be multiple redundant and synergistic circuits composed of different combinations of neural and AEC factors.

\section{References}

Albert P, Boilly B, Courty J, Barritault D 1987 Stimulation in cell culture of mesenchymal cells of newt limb blastemas by EDGF I or II (basic or acidic FGF). Cell Diff 21:63-68.

Albert P, Boilly 1988 Effect of transferrin on amphibian limb regeneration: a blastema cell culture study. Roux's Archives Dev Biol 197:193-196.

Athippozhy A, Lehrberg J, Monaghan JR, Gardiner DM, Voss SR 2014 Characterization of in vitro transcriptional responses of dorsal root ganglia cultured in the presence and absence of blastema cells from regenerating salamanser limbs. Regeneration 1:1-10.

Bauduin B, Lasalle B, Boilly B 2000 Stimulation of axon growth from the spinal cord by a regenerating limb blastema in newts. Brain Res Dev Brain Res 119: 47-54.

This article is protected by copyright. All rights reserved. 
Boilly B, Bauduin B 1988 Production in vitro by spinal cord of growth factor(s) acting on on newt limb regeneration: influence of regeneration of the nerve fibers. Brain Res 466: 155-160.

Boilly B, Cavanaugh KP, Hondermarck H, et al 1991 Acidic fibroblast growth factor is present in regenerating limb blastemas of axolotls and binds specifically to blastema tissues. Dev Biol 145:302310.

Boilly B, Faulkner S, Jobling P, Hondermarck H 2017 Nerve dependence: From regeneration to cancer. Cancer Cell 31: 342-354.

Brockes JP 1984 Mitogenic growth factors and nerve dependence of limb regeneration. Science 235:1280-1287.

Brockes JP, Kintner CR 1986 Glial growth factor and nerve-dependent proliferation in in the regeneration blastema of urodele amphibians. Cell 45:301-306.

Campbell L, Suarez-Castillo EC, Ortiz-Zuazaga H, Knapp D, Tanaka EM, Crews 2011 Gene expression profile of the regeneration epithelium during axolotl limb regeneration. Dev Dynam 240:1826-1840.

Charpentier E, Doudna JA 2013 Rewriting a genome. Nature 495:50-51.

Chew K, Cameron JA 1983 Increase in mitotic activity of regenerating axolotl limbs by growth factorimpregnated implants. J Exp Zool 226:325-329.

Choo AZF, Logan DM, Rathbone MP 1978 Nerve trophic effects : an in vitro assay of factors involved in regulation of protein synthesis in regenerating amphibian limbs. J Exp Zool 206:347-354.

Christensen RN, Weinstein M, Tassava RA 2001 Fibroblast growth factors in regenerating limbs of Ambystoma: cloning and semi-quantitative RT-PCR expression studies. J Exp Zool 290:529-540.

Christensen RN, Weinstein M, Tassava RA 2002 Expression of fibroblast growth factors 4,8,and 10 in limbs, flanks, and blastemas of Ambystoma. Dev Dynam 223:193-203.

This article is protected by copyright. All rights reserved. 
Clark RAF 1996 Wound repair: Overview and general considerations. In: Clark RAF (ed), Molecular and Cellular Biology of Wound Repair. Plenum Press, New York, pp 3-50.

Davies AM 2000 Neurotrophins: neurotrophic modulation of neurite growth. Current Biology 10:R198-R200.

Dmetrichuk JM, Spencer G, Carlone RL 2005 Retinoic acid-dependent attraction of adut spinal cord axons towards regenerating newt limb blastemas in vitro. Dev Biol 281112-120.

Dinsmore CE 1991 Lazzaro Spallazani: concepts of generation and regeneration. In: CE Dinsmore (Ed). A History of Regeneration Research. Cambridge University Press, Cambridge UK, pp 67-90.

Dinsmore CE 1998 Conceptual foundations of metamorphosis and regeneration:from historical links to common mechanisms. Wound Rep Reg 6:291-301.

Dresden M 1969 Denervation effects on newt limb regeneration: DNA, RNA, and protein syhthesis. Dev Biol 19:311-320.

Endo T, Bryant SV, Gardiner DM 2004 A stepwise model system for limb regeneration. Dev Biol 270: 135-45.

Farkas JE, Freitas PD, Bryant DM, Whited JL, Monaghan JR 2016 Neuregulin-1 signaling is essential for nerve-dependent axolotl limb regeneration. Development 143: 2724-2731.

Fei J-F, Schuez M, Tazaki A, et al 2014 CRISPR-mediated genomic deletion of Sox 2 in the axolotl shows a requirement in spinal cord neural stem cell amplification during tail regeneration. Stem Cell Reps 3:444-459.

Fei J-F, Knapp D, Schuez M, et al 2016 Tissue-and time-directed electroporation of Cas9 proteingRNA complexes in vivo yields efficient multigene knockout for studying gene function in regeneration. Npj Reg Med 1: 16002.

This article is protected by copyright. All rights reserved. 
Flowers GP, Timberlake AT, Mclean KC, et al 2014 Highly efficient targeted mutagenesis in axolotl using Cas9 RNA-guided nuclease. Development 141:1-7.

Fu SY, Gordon T 1997 The cellular and molecular basis of peripheral nerve regeneration. Mol Neurobiol 14: 67-116.

Gilbert SF, Barresi MJF 2016 Developmental Biology $11^{\text {th }}$ ed. Sinauer Associates, Sunderland MA.

Globus M, Vethamany-Globus S, Lee YCl 1980 Effect of apical epidermal cap on mitotic cycle and cartilage differentiation in regeneration blastemata in the newt, Notophthalmus viridescens. Dev Biol 75:358-372.

Globus M, Alles P 1990 A search for immunoreactive substance $P$ and other neural peptides in the limb regenerate of the newt Notophthalmus viridescens. J Exp Zool 254:165-176.

Globus M, Smith MJ, Vtehamany-Globus 1991 Evidence supporting a mitogenic role for substance $P$ in amphibian limb regeneration. Involvement of the inisotol phospholipid pathway. Ann New York Acad Sci 632:396-399.

Goldhamer DJ, Tassava RA 1987 An analysis of proliferative activity in innervated and denervated forelimb regenerates of the newt Notophthalmus viridescens. Development 100:619-628.

Han M-J, An J-Y, Kim W-S 2001 Expression patterns of Fgf-8 during development and limb regeneration of the axolotl. Dev Dynam 220:40-48.

Kragl M, Knapp D, Nacu E, Khattak S, Maden M, Henning, et al 2009 Cells keep a memory of their tissue origin during axolotl limb regeneration. Nature 460:60-65.

Kumar A, Brockes JP 2007 Molecular basis for the nerve dependence of limb regeneration in an adult vertebrate. Science 318:772-777.

This article is protected by copyright. All rights reserved. 
Kumar A, Delgado J-P, Gates PB, et al 2011 The aneurogenic limb identifies developmental cell interactions underlying vertebrate limb regeneration. Proc Natl Acad Sci USA 108:13588-13593.

Kumar A, Brockes JP 2012 Nerve dependence in tissue, organ, and appendage regeneration. Neurosci 35:691-699.

Law AJ , Shannon-Weickert C, Hyde TM, Kleinman JE, Harrison PJ 2004 Neuregulin-1 (NRG-1) mRNA and protein in the adult human brain. Neurosci 127:125-136.

Lebowitz P, Singer M 1970 Neurotrophic control of protein synthesis in the regenerating limb of the newt Triturus. Nature 225:824-827.

Looso M, Preussner J, Sousounis K, Bruckskotten M, Michel CS, Lignelli E, et al 2013 A de novo assembly of the newt transcriptome combined with proteomic validation identifies new protein families expressed during tissue regeneration. Genome Biol 14:R16.

Loyd RM, Tassava RA 1980 DNA synthesis and mitosis in adult newt limbs following amputation and insertion into the body cavity. J Exp Zool 214:61-69.

Makanae A, Mitogawa K, Satoh A 2014 Co-operative Bmp-and Fgf-signaling inputs convert skin wound lealing to limb formation in urodele amphibians. Dev Biol 396:57-66.

Manson J, Tassava RA, Nishikawara M 1976 Denervation effects on aspartate carbamyl transferase, thymidine kinase, and uridine kinase activities in newt regenerates. Dev Biol 50:109-121.

McCusker CD, Diaz-Castillo C, Sosnik J, Gardiner DM 2016 Cartilage and bone cells do not participate in skeletal regeneration in Ambystoma mexicanum limbs. Dev Biol 416:26-33.

Meda F, Rampon C, Dupont, et al 2017 Nerves, $\mathrm{H}_{2} \mathrm{O}_{2}$ and Shh: Three players in the game of regeneration. Sem in Cell Dev Biol 80:65-73.

This article is protected by copyright. All rights reserved. 
Mescher AL 1976 Effects on adult newt limb regeneration of partial and complete skin flaps over the amputation surface. J Exp Zool 195:117-128.

Mescher AL 2017 Macrophages and fibroblasts during inflammation and tissue repair in models of organ regeneration. Regeneration 4:39-53.

Mescher AL, Tassava RA 1976 Denervation effects on DNA replication and mitosis during the initiation of limb regeneration in adult newts. Dev Biol 44: 187-197.

Mescher AL, Munaim SL 1986 Changes in the extracellular matrix and glycosaminoglycan synthesis during the initiation of regeneration in adult newt forelimbs. Anat Rec 214:424-431.

Mescher AL, Kiffmeyer WR 1992 Axonal release of transferrin in peripheral nerves of axolotls during regeneration. In: CH Taban, B Boilly (Eds). Keys for Regeneration: Monographs in Developmental Biology 23:100-109.

Mescher AL, Connell E, Hsu C, Patel C, Overton B 1997 Transferrin is necessary and sufficient for the neural effect on growth in amphibian limb regeneration blastemas. Dev Growth Diff 39:677-684.

Mescher AL, White GW, Brokaw JJ 2000 Apoptosis in regenerating and denervated nonregenerating urodele forelimbs. Wound Rep Reg 8:110-116.

Monaghan JR, Epp LG, Putta S, Page RB, Walker JA, Beachy CK 2009 Microarray and cDNA sequence analysis of transcription during nerve-dependent limb regeneration. BioMed Central Biol 7:1.

Mullen LM, Bryant SV, Torok MA, Blumberg B, Gardiner DM 1996 Nerve dependency of regeneration: the role of Distal-less and FGF signaling in amphibian limb regeneration. Development 122:3487-3497.

Muneoka K, Fox WF, Bryant SV 1986 Cellular contribution from dermis and cartilage to the regenerating limb blastama in axolotls. Dev Biol 116: 256-260.

This article is protected by copyright. All rights reserved. 
Nowoshilow S, Schloissnig S, Fei J-F, et al 2018 The axolotl genome and the evolution of key tissue formation regulators. Nature 554: 50-55.

Pirotte N, Leynen N, Artois T, Smeets K 2015 Do you have the nerves to regenerate? The importance of neural signaling in the regeneration process. Dev Biol 409:4-15

Poulin ML, Patrie KM, Botelho MJ, Tassava RA, Chiu I-M 1993 Heterogeneity in expression of fibroblast growth factor receptors during limb regeneration in newts (Notophthalmus viridescens). Development 119:353-361.

Poulin ML Chiu I-M 1995 Re-programming of expression of the KGFR and bek variants of fibroblast growth factor receptor 2 during limb regeneration in newts (Notopthalmus viridescens). Dev Dynam 202:378-387.

Rao N, Jhamb D, Milner DJ, Li B, Song F, Wang M, et al 2009 Proteomic analysis of blastema formation in regenerating axolotl limbs. BioMed Central Biol 7: 83

Rao N, Song F, Jhamb D, Wang M, Milner DJ, Price NM, et al 2014 Proteomic analysis of fibroblastema formation in regenerating hind limbs of Xenopus laevis froglets and comparison to axolotl. BioMed Central Dev Biol 14:32.

Richmond MJ, Pollack ED 1983 Regulation of tadpole spinal nerve growth by the regenerating limb blastema in tissue culture. J Exp Zool 225:233-242.

Sandoval-Guzman T, Wang H, Khattak S, Schuez M, Roensch K, Nacu E, et al 2014 Fundamental differences in dedifferentiation and stem cell recruitment during skeletal regeneration in two salamander species. Cell Stem Cell 14:174-187.

Satoh A, Makanae A, Nishimoto Y, Mitogawa K 2016 FGF and BMP derived from dorsal root ganglia regulateblastema induction in limb regeneration in Ambystoma mexicanum. Dev Biol 417:114-125.

Sidman RL, Singer M 1960 Limb regeneration without innervation of the apical epidermis in the adult newt, Triturus. J Exp Zool 144:105-11.

This article is protected by copyright. All rights reserved. 
Singer M 1952 The influence of the nerve in regeneration of the amphibian extremity. Quart Rev Biol 27:169-200.

Singer M 1964 The trophic quality of the neuron: some theoretical consideration. Progress in Brain Res 13:228-232.

Singer M 1965 A theory of the trophic nervous control of amphibian limb regeneration, including a re-evaluation of of quantitative nerve requirements (pp20-32). In: V Kiortsis, HAL Trampisch (Eds). Regeneration in Animals and Related Problems. Amsterdam, North-Holland.

Singer M 1978 On the nature of the neurotrophic phenomenon in urodele limb regeneration. Am Zool 18:829-308.

Singer M, Mutterperl E 1963 Nerve fiber requirements for regeneration in forelimb translants of the newt, Triturus. Dev Biol 7:180-191.

Singer M, Ilan J 1977 Nerve-dependent regulation of absolute rates of protein synthesis in newt limb regenerates measurement of methionine specific activity in peptidyl-tRNA of the growing polypeptide chain. Dev Biol 57:174-187.

Smith GN, Toole BP, Gross J 1975 Hyaluronidase activity and glycosaminoglycan synthesis in the amputated newt limb: comparison of denervated non-regenerating limbs with regenerates. Dev Biol 25:849-856.

Smith MJ, Globus M 1989 Multiple interactions in juxtaposed monolayer of amphibian neuronal, epidermal, and mesodermal limb blastema cells. In Vitro Cell and Dev Biol 25:849-856.

Stocum DL 1975 Outgrowth and pattern formation during limb ontogeny and regeneration. Differentiation 3:167-182.

Stocum DL 1995 Wound Repair, Regeneration and Artificial Tissues. RG Landes, Austin, TX.

This article is protected by copyright. All rights reserved. 
Stocum DL 2011 The role of peripheral nerves in urodele limb regeneration. Eur J Neurosci 34:908916.

Stocum DL 2017 Mechanisms of urodele limb regeneration. Regeneration 4: 159-200.

Stocum DL, Dearlove GE 1972 Epidermal-mesodermal interaction during morphogenesis of the limb regeneration blastema in larval salamanders. J Exp Zool 181:49-62.

Stocum DL, Cameron JA 2011 Looking proximally and distally: 100 years of limb regeneration. Dev Dynam 240:943-968.

Tassava RA, Mescher AL 1975 The roles of injury, nerves and the wound epidermis during the initiation of amphibian limb regeneration. Differentiation 4:23-24.

Tassava RA, McCullough WD 1978 Neural control of cell cycle events in regenerating salamander limbs. Am Zool 18:843-854.

Thornton CS 1960 Regeneration of asensory limbs of Amblystoma larvae. Copeia 4:371-373.

Thornton CS 1968 Amphibian limb regeneration. Adv Morphog 7:205-249.

Thornton CS, Thornton MT 1970 Recuperation of regeneration in denervated limbs of Ambystoma larvae. J Exp Zool 173:293-301.

Tonge DA, Leclere G 2000 Directed axonal growth towards axolotl limb blastemas in vitro. Neurosci 100:201-211.

Voss SR, Palumbo A, Nagarajan, Gardiner DM, Muneoka K, Stromberg AJ, et al 2015 Gene expression during the first 28 days of axolotl limb regeneration:Experimental design and global analysis of gene expression. Regeneration 2: 120-36

This article is protected by copyright. All rights reserved. 
Wang L, Marchionni MA, Tassava RA 2000 Cloning and neuronal expression of a type III newt neuregulin and rescue of denervated nerve-dependent newt limb blastemas by rhGGF2. J Neurobiol 43:150-158.

Yntema CL 1959a Regeneration of sparsely innervated and aneurogenic forelimbs of Ambystoma larvae. J Exp Zool 140:101-123.

Yntema CL 1959b Blastema formation in sparsely innervated and aneurogenic forelimbs in Amblystoma larvae. J Exp Zool 142:423-440.

Yun M, Davaapil H, Brockes JP 2015 Recurrent turnover of senescent cells during regeneration of a complex structure. eLife:4:e05505 DOI 10.7554/eLife.05505.

\section{Legends for Figures}

Fig. 1: Summary of blastema formation, which results in the formation of an accumulation blastema, and development, which involves growth and patterning of the blastema. This phase can be divided into several morphological stages, a conical medium bud, a larger late bud initiating cartilage differentiation, the emergence of fingerbuds and growth to complete regeneration. A-D, longitudinal sections through (A) the accumulation blastema stage, (B) the medium bud stage, (C), the late bud stage, $(D$, the two-fingerbud stage. $E$, $F$, whole mounts stained for cartilage with methylene blue. E, two-fingerbud stage; $F$, the finished regenerate. Reproduced from Stocum, Mechanisms of urodele limb regeneration. Regeneration 4: 159-200, Open Access.

Fig. 2: Reciprocal relationship between blastema (b) and nerves (black lines and arrows). Limb amputated through the mid-stylopodium of forelimb (s). (A) As the blastema forms, it produces factors (yellow arrow) that promote axon regeneration. (B) Regenerating axons provide neurotrophic factors that promote blastema growth and result in the regeneration of a normal limb. $\mathrm{z}=$ zeugopodium; c = carpals. (C) Denervation ( $\mathrm{x}$ ) at medium bud stage does not inhibit differentiation and morphogenesis of the blastema, but mitosis is inhibited and the blastema forms a miniature regenerate.

This article is protected by copyright. All rights reserved. 
Fig. 3: Experiments by Globus, et al, Dev Biol 75:358-372, 1980, demonstrating effect of the AEC on mitosis of blastema cells. (A) A medium bud stage blastema (red) implanted deep in a dorsal fin (DF) tunnel forms a miniature, distally truncated regenerate, whereas the same stage blastema implanted so that its distal tip protrudes from the tunnel and is re-covered by fin epidermis (yellow line) forms a PD-complete miniature regenerate. $V C=$ vertebral column; $V F=$ ventral fin. (B) Blastemas cultured on the opposite side of a nanopore filter (dashed red line) from spinal ganglia (gold). Left, blastema mesenchyme (blue) with AEC (green). The blastema cells undergo mitosis. Right, blastema mesenchyme without AEC. The blastema cells withdraw from the cell cycle and differentiate into chondrocytes.

Fig. 4: Models of functional synergy between nerve, AEC, and blastema cells. (A) The AEC (yellow) produces factors (black arrows) that maintain the blastema cells (blue) in an undifferentiated state. The nerve produces separate factors (red arrow) that stimulate mitosis of the blastema cells. (B) The AEC produces factors necessary to maintain the undifferentiated state of the blastema cells, but depends on nerve factors to carry out this function. (C) The nerve, AEC and the blastema cells themselves all produce the same factors (red arrows, red dots).

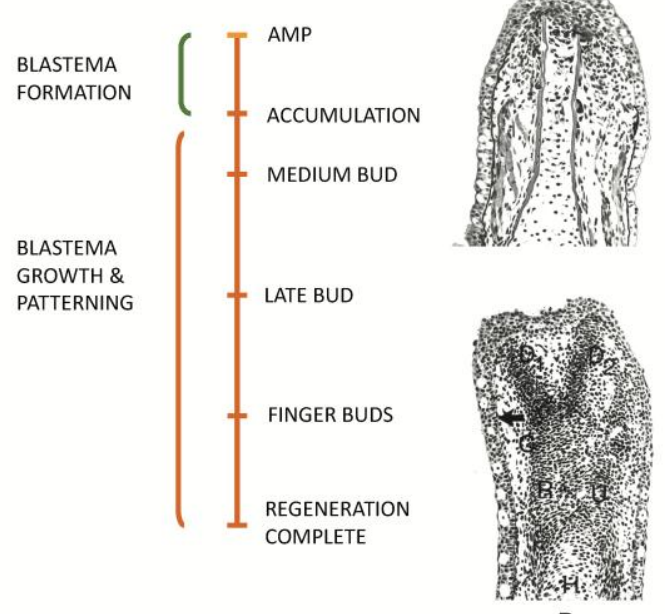

D
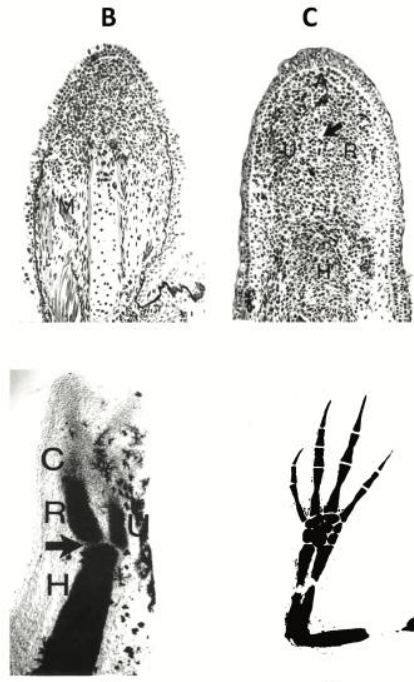

$\mathbf{E}$

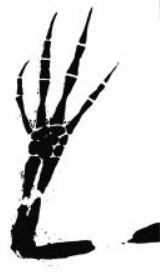

$\mathbf{F}$

This article is protected by copyright. All rights reserved. 


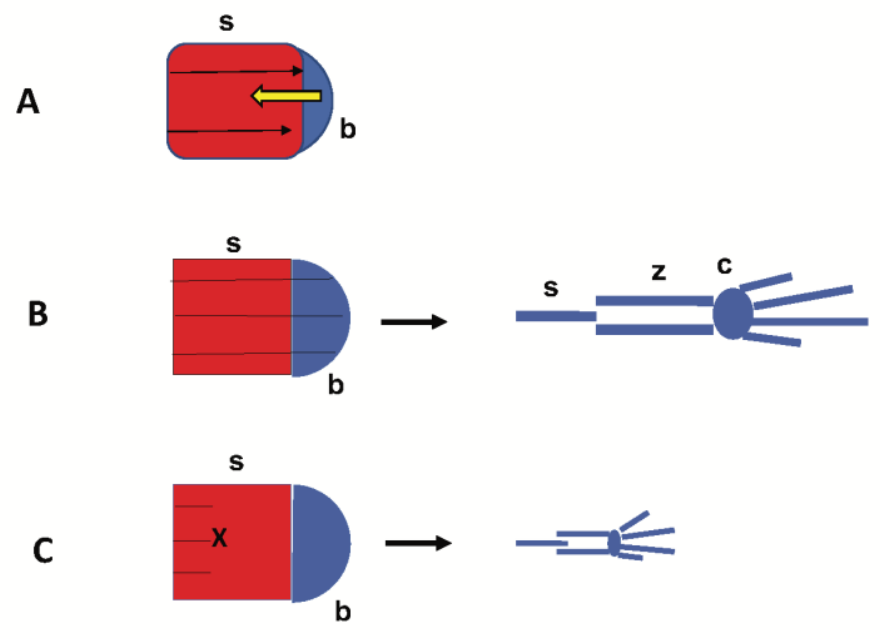

This article is protected by copyright. All rights reserved. 
A

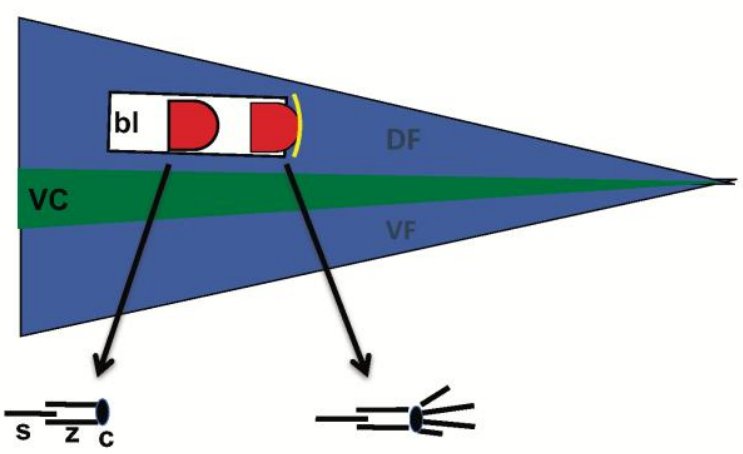

B

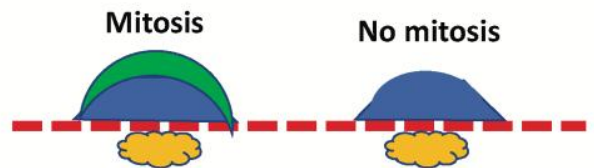

This article is protected by copyright. All rights reserved. 
A Nerve, AEC express

Different factors that Have different roles in Cell cycle

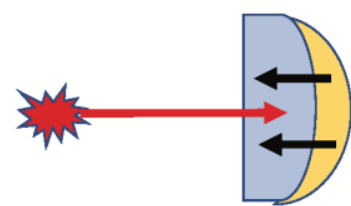

B Nerve stimulates Expression of AEC Mitogenic factor(s)

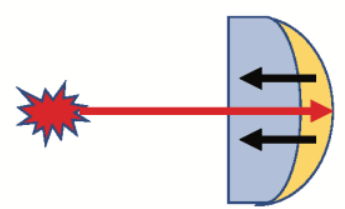

C Nerve, AEC and Blastema cells express Same mitogenic factor

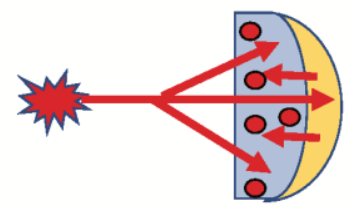

This article is protected by copyright. All rights reserved. 